\title{
LES RELATIONS ECONOMIQUES INTERNATIONALES
}

\section{Carney}

Deuxième leçon : La théorie moderne du commerce international - I'offre et la Demande.

I. La réponse moderne aux quatre questions posées par les économistes classiques.

a) Raisons de l'échange : différences des coûts absolus, des facteurs de production et des prix absolus des prorluits.

Du côtté de l'offre : (i) La différence des coûts de production reflète les différences dans la disponibilité des facteurs et dans les prix des facteurs.

(ii) Les coefficients des coûts ne sont pas identiques (en gros, la théorie des coûts comparatifs de Ricardo, mais en évaluant la production par référence à la monnaie plutôt qu'en termes de travail).

Notez bien

: (1) Tous les types de variations des coûts sont inclus: constants, croissants et décroissants, et non pas seulement les coûts constants comme dans la théorie classique.

(2) La différence entre les coûts des facteurs d'un pays à l'autre reflète aussi parfois des modifications (et non pas seulement des fluctuations du marché) dans les taux de l'échange et les coûts des transports.

Du côté de la demande : a)Les différences entre les prix des produits d'un pays à l'autre sont fondées sur les différences dans les facteurs de production dont sont dotés les pays, et dans la demande intérieure. 
b) Quels sont les biens produits et échangés : ceux qui étant donné les différences de coùt absolu, offrent des avantages monétaires comparatifs.

c) Comment sont déterminés les termes de liẹchange et comment les gains sont-ils répartis : denande réciproque, même réponse que celle de Mill.

d) Comment se maintient l'équilibre de la balance commerciale et des paiements a dans I'hypothèse du papier-monnaie et duntaux de change libre, l'équilibre est atteint grâce à des ajustements du taux de change, alors que les prix intérieurs restent invariables.

II. Incidence du commerce international sur le prix des facteurs.

Lorsque res facteurs sont immobiles maị que les produits sont mobiles :

Vì les mêmes şchémas que pour la section III (a) Si les coûts sont constants, pas d'incidence sur le prix des facteurs:

(b) Si les coûts sont croissants, tendance au nivellement des prix des facteurs:

(c) Si les coûts sont décroissants, les écarts entre les prix des facteurs ont tendance à s'accrô̂tre.

III. Incidence des variations des coûts sur la production.

(a) Coûts constants - spécialisation complètc

(b) Coûts croissants - spécialisation partielle

(c) Coûts décroissants - spécialisation complète (historique)

IV. Incidence du coût des transports sur le commerce international.

(1) Il diminue les différences de coût entre les régions et entre les pays.

(2) Il influence le lieu d'implantation des industries en les encourageant à s'installer dans les régions où le coût des facteurs est le plus bas possible.

Le prix des transports joue uh rôle important dans la localisation des industries :

(a) qui sont orientées vers les matières premières

(b) qui sont orientées vers les marchés

(c) qui sont neutres ou "sans entraves". 
V. Le revenu national et le commerce extérieur.

Par le mécanisme du multiplicateur et de l'accélérateur du commerce extérieur, les exportations produisent des revenus, et les importations les détruisent.

Objections à la doctrine du multiplicateur du commerce extérieur : (1) La conclusion logique serait que pour arriver à l'incidence maximum sur les revenus, les exportations devraient être augmentées et les importations réduites au minimum. Il s'agit là de la version de la doctrine mercantiliste à propos des biens-réels. Th fait, la doctrine du multiplicateur est mercantiliste, comme l'a clairement expliqué Keynes, et par consécuent, très nationaliste et applicable seulement à des cas particuliens de pays où la demande globale est insuffisante.

(2) Cette doctrine est contraire au commerce international car elle suppose une économie fermêe, icndis que le commerce international suppose une économie ouv rte. Flle $n ' a$ dono pas sa place dans les relations économiques internationales.

(3) Il n'est en général ras réalisable dans la pratique que tous les pays adoptent simultanémen; une politique d'accumulation des excédent d'exportations pour a'gmenter l uurs revenus nationaux.

(4) Une doctrine du reven national et du commerce international qui soit juste doit être fondé sur le postulat d'une économie ouverte et non pas d'une économie fernée; c'est-ì-dire, que si dans une économie ouverte les importatiors sont la clef de voûte des investissements, l'accroissement des exrortations et de l'épargne en devises peut abaisser le niveau du rəvenu national alors que l'augmentation des importations, et en pariculier des importations de biens d'équipement des épargnes en devises peut le relever.

VI. Les défauts des théories clessiques et modernes du commerce international. La théorie olassique incorpore le commerce extérieur à la théorie générale du niveau des prix, la théorie moderne incorpore le commerce au revenu national ou productior totale; mais ni l'une ni l'autre n'incorporent explicitement lo commerce à la fois à la théorie générale du niveau des prix, et à la théorie de la production globale. Le résultat obtenu par Keynes en incorporent la production à la théorie générale des prix n'a pas encore fait sentir son influence sur la théorie moderne $d v$. commerce international. 
VII. Notez bien :

La théorie moderne du commerce international n'est autre que la mise en application de la théorie moderne de la valeur au domaine du commerce extérieur. Ainsi, la doctrine moderne incorpore l'échange international aux travaux d'Eli Heckscher de l'Université de Stockholm (L'incidence du commerce extérieur sur la distribution du revenu, Ekonomick Tidskrift, 1919) et de Bertil Ohlin (Commerce Interregional et International, Harvard University Press, Cambridge 1933). Les travaux d'Ọlin ont paru au moment où Joan Robinson et Edward Chamberlin formulaient et répandaient les doctrines de la concurrence imparfaite et de la concurrence monopolistique. Ces doctrines faisaient les plus expresses réserves sur la traditionnelle doctri. . classiquo généralement, acceptée de la concurrence parfaite, des prix uniformes et de l'équilibre à long terme. Il $n^{\prime}$ est donc pas étonnant que la doctrine moderne du commerce international ait été fortement influencée par les doctrines remaniées de la valeur et de la détermination des prix.

Dakar, 26 Février 1964. 


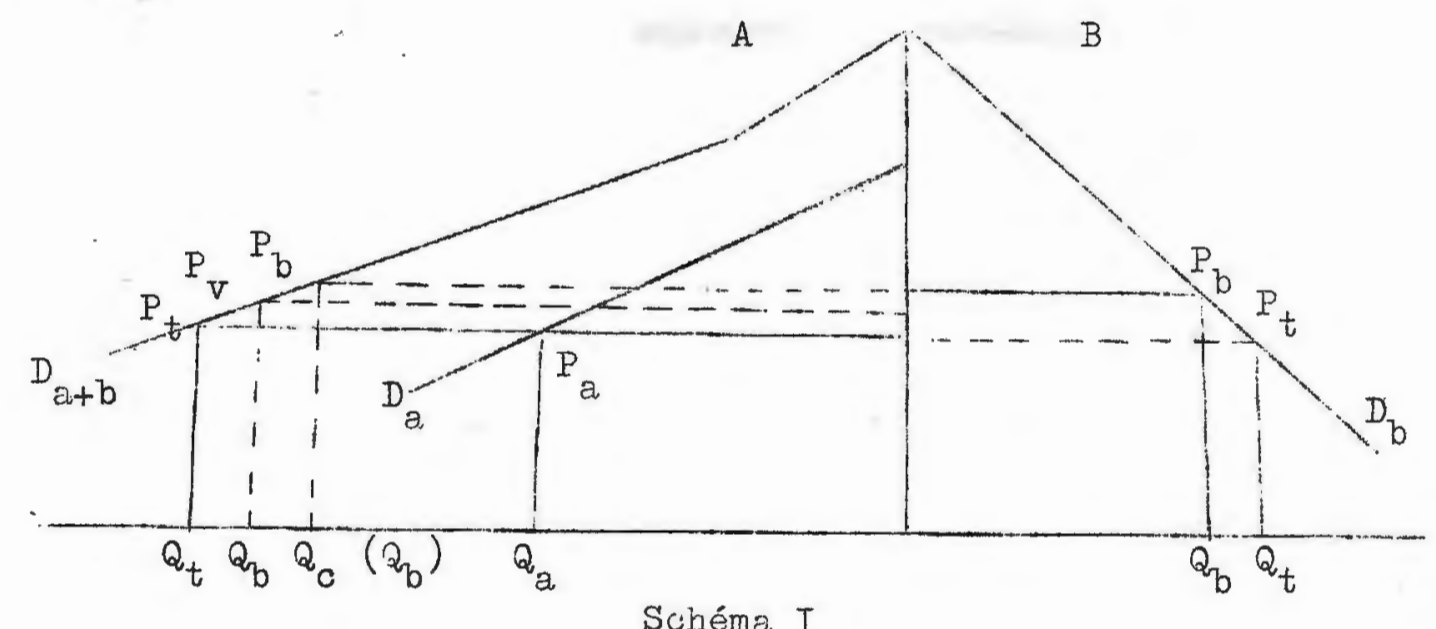

Differentielles des coûts, si les coûts de production sont constants. Cas de spécialisation complète.

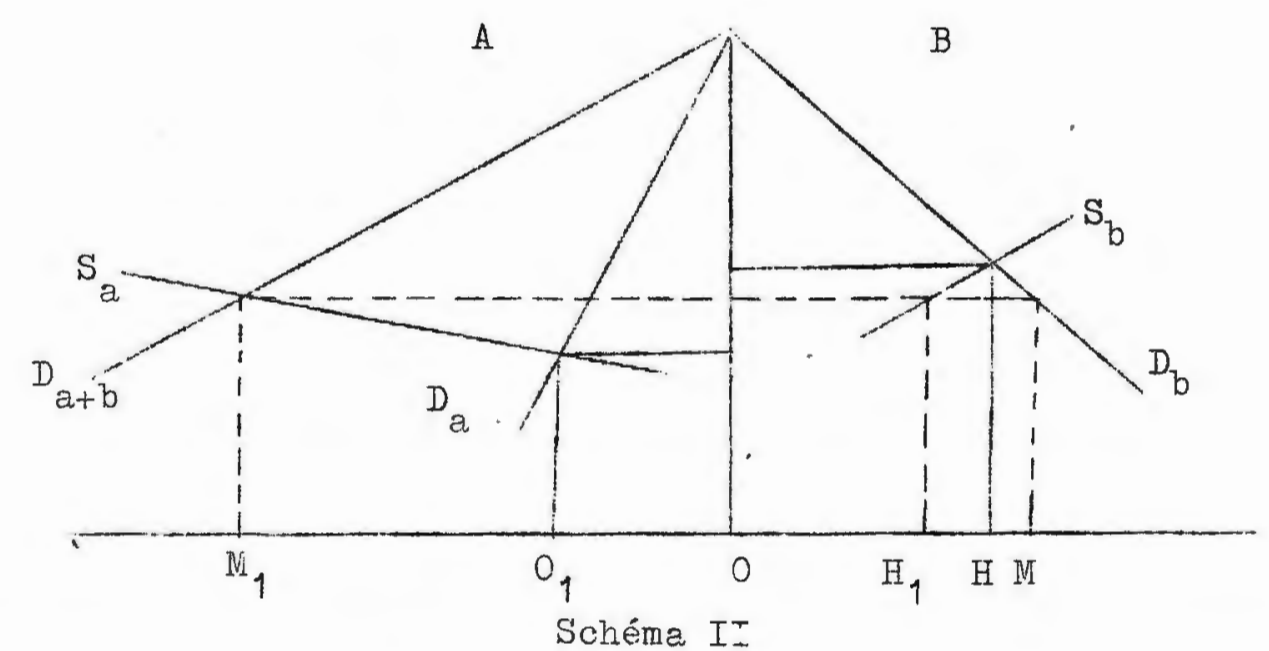

Différentielles des coûts avec les coûts de production croissants Cas de spécialisation partielle.

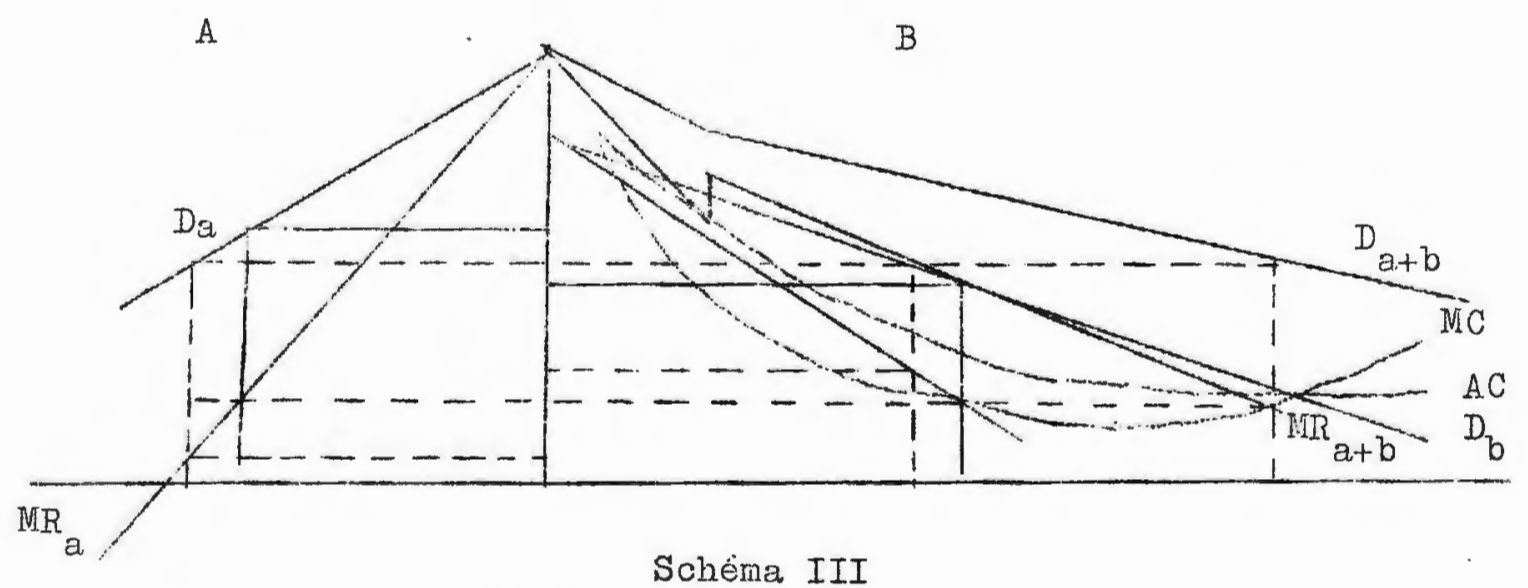

Coûts décroissants: Spécialisation complète 
NATIONS UNIES

INSTITUT AFRICAIN

DE TI WLOPPEMENT ECONOMIQUE

FT DE PLANIFICATION

DA KA R
IDEP/ET/VII/107

Leçons 3 et 4

Original : anglais

Avril 1964

RELATIONS ECONOMIQUES INTERNATIONAIES

(Troisième et quatrième leçon)

D. Carney

Troisième lecon : Concurrence parfaite, Monopole et concurrence monopolitique: leur incidence sur le commerce international.

Comme on l'a vu dans la leçon précédente, la théorie moderne au commerce international n'est autre qu'une application de la théorie moderne de la valeur au domaine du commerce internationel. La présente leçon porte sur les différents types de concurrence du marché et leur incidence sur le commerce extérieur. Elle constitue dono un moyen commode de passer en revue les éléments de la théorie de la valeur et également une occasion d'appliquer la théorie aux problèmes du commerce extérieur.

$$
-:-:-
$$

Quatrième leçon : Ia balance commerciale et la balance des paiements. La balance des paiements: comprend :

Toutes les opérations qui doivent être réglées dans la période courante (c'est-à-dire en un an), telles que les importations, los exportations, Ies services ce frêt, etc.), et qui impliquent des mouvements de capitaux à court terme vu que le paiement se fait habituellement par des dépôts dans les banques locales ou étrangères. Cependant, pour les besoins de la balance des paiements toutes ces opérations sont inscrites au COMPTE COURANT : côté passif slil s'agit de payer et côté actif s'il s'agit d'être payé. Le solde net de toutes ces opérations est ce qu'on appelle un mouvoment de capital à count terme; il est inscrit au COMPTE CAPITAI dans la colonne appropriée. Elle est alors considérée comme l'excédent ou déficit du compte courant. 
Mouvements de capitaux à court terme.

Les mouvements de capitaux à court terme dans la balance des paiements tombent dans deux catégories :

(a) Ceux qui sont le résultat net (excédent ou déficit) d'opérations inscrites au compte courant.

(b) Ceux qui sont entrepris délibérément pour eux-mêmes :

(i) Pour augmenter le revenu : achat de valeurs étrangères à court terme (bons du Trésor, effets de commerce, acceptations bancaires), afin de se procurer un revenu plus élevé que celui qu'on pourrait obtenir chez soi.

(ii) Spésủ atifs : transactions en monnaies étrangères (accumulation de réserves en devises) en anticipation des modifications qui pourraient se produire quant à la valeur internationale, (cest-à-dire/taux du change officiel) de telle ou telle monnaie.

(iii) Provoqués par l'incertitude : transferts des avoirs financiers à l'étranger par crainte de guerre, d'instabilité politique ou d'inflation.

Note : La balance dəs paiements :

Montre : 1. I'endettement courant et le mode de financement do l'excédent du déficit.

2. Les balances de plusieurs années consécutives sont très révélatrices en ce qui concerne le caractère et l'ampleur des modifications de la balance des paiements et ses tendances.

Elle ne montre pas :

1. Si l'amlioration ou la détérioration est due à des changements.

(a) Dans la politique commerciale

(b) Dans les taux de change (par ex. - dépréciation ou revaĺorisation)

(c) Relativement à quoi et.à qui l'excédent ou le déficit se sont produits. 
2. Le solde de la dette globale, mais seulement les modifications courantes nettes dans la balance de la dette.

3. Si les changements sont dus à un déséquilibre structurel ou bien à des causes temporaires telles qu'une mauvaise récolte/des transferts spéculatifs de fonds. C'est-à-dire n'indique pas pourquoi le changement s'est produit.

4. Ne dit rien à propos de la politique commerciale du pays qui pourrait être à la base du déficit ou de l'excédent.

5. Ne montre pas si le déséquilibre fondamental est un aspect du changement dans les relations créditeur-débiteur d'un pays, c'est-àdire, s'il est en train de passer par les quatre grands stades de I'évolution de la balance des paiements et de l'endettement.

Dakar

Conférence donnée en Mars 1964. 
$-.$.

$\cdots$

$\therefore$ 
NATIONS UNIES

INSTITUT AFRICAIN

DE DEVELOPPEMENT ECONOMIQUE

ET DE PLANIFICATION

D A K A R
IDEP/ET/VII/ 107

Leçon 5

Original : anglais

Avril 1964

\section{RELATIONS ECONONIQUES INTERNATIONALES}

\section{Carney}

Cinquième leçon : Le marché des devisos et les accords internationaux.

A. - 1. Moyens de règlement du commerce:) Les importations servent en génẻre. ffets de commerce (étalon or). ) à règler lé exportations par

) transfert des créancos entre les

) importateurs et les exportateurs

) locaux (plus les flux de l'or

) dans le sys tème de l'étälon-or).

Transferts télégraphiques (mo- )

derne).

Lettre de crédit.

2. Arbitrage - égaliser les différences dans les taux du change.

3. Marché à terme (couverture) pour se prémunir contre les risques du changement de valeur de la monnaie au momont du paiement (ces

transactions à terme peuvent tendre soit à stabiliser, soit à

déséquilibrer le marché suivant que les partios pensent que le sens du changement de valeur de la monnaie pourra ou non être inversé.

B. Règlement dans différents sytèmes du taux de change.

I. Taux de change fixe (étalon or)

Ajustement par :

1. Changement dans le niveau des prix et des revenus.

2. Renversement de la direction du'flux des échanges.

II. Taux de change variable.

Ajustement par :

1. Changement du taux de change

2. Renversement du flux des fonds (à long terme)

Note: Pas de changement pour les prix et le nịveau des revenus extérieurs. 
3. Substitution de produits nationaux aux produits importés
4. (t arbitrage
(+ flux spéculatifs des fonds)
à court terme

5. Dépréciation du change à condition que l'élasticité des exportations soit supérieure à 1

III. Contrôle des changes.

Ajustement par le :

1. Contrôle du volume des exportations et des importations pour les contraindre à I'équilibre : (a) licences d'exportations

(b) contingentements (c) centralisation des devises.

\section{Dépréciation des changes.}

Pour combler un déficit par la dépréciation du taux de change, il faut passer par les étapes suivantes :

(1) La dépréciation peut réduire les importations par rapport aux exportations et ainsi diminuer la demande de devises étrangères.

(2) La dépréciation peut permettre d'abaisser los prix des exportations et si l'élasticité de la demande étrangère pour les exportations est plus grande que l'unité $(\varepsilon>\mid)$ pour un seul pays dépréciateur, ceci peut activer les ventes et augmenter les réserves de devises étrangères.

(3) Un large Éventail de marchandises locales peut être écoulé sur le marché de l'exportation, c'est-à-dire de marchandises aptes à l'exportation du fait de la dépréciation du taux de change et de l'abaissement du prix des exportations, augmentant ainsi l'offre en devises étrangères.

(4) Tous ces résultats peuvent se produire dans les cas (1) à (3), à condition qu'il n'y ait pas de dêpréciation comparable du taux de change dans un autre pays.

Par conséquent, les conditions dans lesquelles on peut employer utilement la méthode de la dépréciation du taux de change pour éliminer le déficit de la balance des paiements sont les suivantes :
(a) $\mathcal{E}>\mid$ en ce qui concerne les demandes extérieures d'exporta- tions. 
(b) il ne faut pas dévier les exportations du marché extérieur, vers le marché intérieur, dont les prix sont plus élevés.

(c) pas de dépréciation concurrentielle du taux de change à l'étranger.

(d) le commerce extérieur du pays qui déprécie le taux de change ne doit représenter qu'une proportion relativement minime du commerce mondial total, sinon on ne tirerait aucun bénéfice de la dépréciation du taux de change, car dans ce cas il serait difficile que. soit plus grand que l'unité pour la demande extérieure d'exportations, et il serait difficile pour le flux des produits exportables d'augmenter de volume sur le marché de I'exportation.

Remarque : Fonds de régularisation des changes.

Il s'agit là d'une conséquence de l'étalon or qui s'est développé après son abandon dans les années 30. Il s'agissait d'un fonds qui avait été créé pour éviter que les mouvements spéculatifs de fonds ne déprécient ou au contraire n'augmentent la valeur extérieure de la monnaie.

DAKAR

Conférence donnée en Mars 1964. 
$-$

? 
- NATIONS UNIES

l. INSTITUT AFRICAIN

IDEP/ET/VII/ 107

DEVELOPPEMENT ECONOMIQUE

ET DE PLANIFICATION

DAKAR

Leçon 6

Original : anglais

Avril 1964

D. Carney

\section{RELATIONS ECONOMIQUES INTERNATIONALES}

Sixième leçon : Revenu national et Commerce extérieur.

Dans nos deux premières leçons. sur les théories classique et moderne de l'échange international on note deux faits saillants au niveau global :

(1) Le commerce extérieur n'affecte pas le volume du produit intérieur mais seulement le niveau des prix. Dans la théorie classique, le commerce extérieur est intégré à la théorie générale du niveau des prix par le mécanisme du flux numéraire. Le produit reste inchangé puisque la-théorie classique du niveau des prix suppose que ie produit total est constant et indépendant du niveau des prix. On effectue des transactions sur le même volume de produits et d'affaires qu'il y ait plus ou moins de monnaie en circulation, la seule incidence du changement du niveau des prix étant (i) des changements dans la valeur de la monnaie et (ii) les transferts de revenu entre les différents groupes économiques de la société.

En ce qui concerne le commerce extérieur, le flux des importations et des exportations prédomine suivant que l'or afflue dans l'économie ou qu'il fuit, de sorte que, si l'on suppose un volume de produit constant, la part des importations dans le revenu total est plus forte que celle des exportations lorsque. Ies prix montent, au moment où l'or afflue et vice versa lorsque les prix baissent au moment où l'or fuit.

Par conséquent, l'adaptetion du revenu total au commerce prend. la forme d'un accroissement de la proportion des importations par rapport au revenu total lorsque les prix montent, et d'un accroissement de la proportion des exportations par rapport au rovenu total lorsque les prix baissent. La seule incidence du commerce extérieur sur le revenu national est donc un changement dans les proportions relatives des importations et des exportations dans le produit total, le produit total restant invariable. 
(2) Selon la théorie moderne, le commerce extérieur est intégré au produit total (ou revenu national) par le truchement des multiplicateurs et des accélérateurs des investissements internes ot du commerce extérieur, dont l'effet total est que $X$ crée $Y$, tandis que M détruit $Y$. Aucune mention particulière n'est faite du changement du niveau des prix, sinon' que ces changements sont neitres ou constants.

I. Economie fermée

$$
\mathrm{C}+\mathrm{I}=\mathrm{Y}=\mathrm{C}+\mathrm{S}
$$

En équilipre $I=S$

MPC + MPS $=1$

Multiplicateur $=K=1 /(1-$ MPC $)=1 /$ MPS $=\frac{d Y}{d I}$

II. Economie ouverte

Introduire les investissements étrangers

$$
c+I_{d}+I_{f}=Y
$$

Lorsque $I_{f}=X-M$

de manière que $\mathrm{C}+\mathrm{I}_{\mathrm{d}}+\mathrm{X}=\mathrm{Y}+\mathrm{N}=\mathrm{C}+\mathrm{S}+\mathrm{M}$

et que $I_{d}+X=S+M$

$\mathrm{Si} I_{\mathrm{d}}+I_{f}=S=0, I_{d}=I_{f}=0$

alors $X=M$ (comme le suppose la doctrine du flux numéraire de Hume)

alors $\frac{d Y}{d X}=\frac{d Y}{d M}=1 / \frac{d M}{d Y}=K$ (multiplicateur du commerce extérieur)

c'est-à-dire le changement de $\mathrm{Y}$ dû au changement de $\mathrm{X}=$ la réciproque de la propension marginale à l'importation. On arriverait au même résultat, si dans le multiplicateur intérieur on remplaçait l'épargne par $M$ et les investissements par $X$.

(1) MPC = Propension marginale à la consommation. MPS = Propension marginale à l'épargne. 
En supposant que $I_{d}$ est constant à tous les niveaux de $Y$ parce que c'est le facteur indépendant dans le système fermé, alors $d X=d S+d M$ et si l'on divise les deux côtés de l'équation en dY,

$$
\begin{aligned}
& \frac{d Y}{d X}=\frac{d Y}{d S+d M}=K_{9} \text { multiplicateur, en tenant compte } d u \text { commerce extérieur } \\
& \left(=\frac{1}{M P S+M P M}\right) \quad K=1 /(1-M P C+N P M)=1 /(1-\overline{M P C-M P M})
\end{aligned}
$$

Dans cette mesure la valeur de dC est réduite, réduisant ainsi la valeur du multiplicateur des investissements chaque fois que les importations sont reçues de l'ortérieur. L'effet multiplicateur des investissements sur $Y$ est le plus grand lorsque dM $=0$ et par conséquent lorsque l'excédent des exportations est investi à l'étranger.

Ainsi, selon cette analyse les exportations sont créatrices de revenu et les importations destructrices de revenu, car ces dernières constituent une fuite hors du circuit de la consommation de la même manièro que l'épargne et les impôts sont considérés comme fuites par l'approche du revenu.

Cette analyse n'ost vraie que fuur uno économie ouverte dans laquelle los investissements locaux ne dépendent pas des importations en provenance de l'étranger. Mais si cette dépendance existait, on aurait le rapport inverse; los importations seraient créatrices de revenu, car elles augmenteraient dC au lieu do dS, et le multiplicateur on tenant compte du commerce extérieur, deviendrait 1/(1-MPC + MPM) avec la réserve que l'économie doit éventuellement être à même de créer sa propre technologie et ses propres industries de biens d'équipement. Sinon, I'économie est simplement greffée sur la civilisation industrielle.

- Notez également que dans toute l'analyso qui précède, l'une des hypothèses fondamentales est que les prix sont constants et qu'il $y$ a des ressources oisives. L'accélérateur du commerce extérieur.

On peut parler d'un effet d'accélérateur dans le secteur du commerce extérieur aussi bien que dans le secteur intérieur, une augmentation des 
exportations aboutissant à un excédent des importations par l'intermédiaire d'une augmentation des investissements. Kindleberger donne deux exemples pour illustrer cette théorie:

(a) Le cas où un accroissement des exportations aboutit à une augmentation des investissements dans les industries d'exportation: par exemple une"augmentation des dépenses des touristes américains à Londres (c'es-à-dire une augmentation des exportations britanniques invisibles) a provoqué la construction à Londres de nouveaux hôtels pour l'industrie touristique, et par là à une augmentation encore plus poussée dos exportations invisibles.

(b) Le cas où une augmentation des exportations provoque de nouvoaux investissoments et une production accrue dans les industries dont les produitó sont destinés à la consommation intérieure. Par exemple, une augmentation des exportations d'arachides du Sénégal aboutit à des investissements sans les usines de transformation en vue de produjre de l'huile d'arachide pour la consommation intérieure.

A la suite de cette étude on peut formuler les critiques suivantes à l'oncontre des théories classique et moderne du commerce international en ce qui concerne les relations entre les théories de l'échange et du revenu national:

(1) Pour ce cui est de la théorie classique elle suppose à tort que le niveau des prix n'a aucun effet sur le niveau du revenu ou de la production et pour cette raison elle n'intègre pas le rovenu national au commerce extérieur. Keynes a changé tout cela dans sa Théorie Générale de l'Emploi, de I'Intérêt et de la Monnaie, où il démontre que l'un des éléments de l'accroissement de la demande monétaire est l'accroissement des prix ainsi que l'augmentation de la production jusqu'à ce qu'on atteigne le plein emploi, niveau où prend effet la théorie quantitative classique de la monnaie: accroissement des dépenses aboutissant simplement à une augmentation inflationniste des prix sans augmentation de la production. 
(2) Quant à la théoric moderne olle suppose à tortque l'effet de multiplicateur du commerce extérieur sur la production intérieure pourrait se produire sans aucune modification du niveau des prix et ceci après ce qui a été écrit par Keynes.

Deuxièmement, que $X$ est créateur de $Y$ alors que $M$ est destructeur de $Y$, quand le contraire pourrait être vrai dans les pays en voie de développement à économie ouverte. Les deux théories ne fournissent, dono qu'une explication partielle puisqu'elles n'établissent pas la relation entre d'une part, le commerce extérieur, le niveau des prix et le niveau du revenu, d'autre part. Troisièmenent, Iorsque la théorie moderno conclut que $X$ est créateur et $\mathbb{M}$ destructeur de revenus, elle affirme une thèse extrêmement naticraliste, mercantiliste et contraire à l'esprit du commerce international orienté vors des gains réciproques. Quatrièmement, de cette même thèse, on devrait conclure que tous les pays auraient intérêt à constituer des excédents d'exportations et à réduire les importations au minimum - ce qui est manifestement impossible, puisque pour qu'un pays exporte, il faut bien qu'un autre pays importe. Cette thèse ne peut done être vraie que pour un seul pays à la fois et non pas pour tous les pays en même temps. Cinquièmement, c'est la thèse inverse qui vaut pour les économies sous-développées ou en voie de développement qui n'ont pas d'industries locales produisant des biens d'équipement et qui n'ont pas leur propre technologie. La thèse est donc'possible en pratique pour un seul pays développé à condition que ce pays ait sa propre technologie et des industries locales d'équipement capables de produire des marchandises locales pour remplacer les produits d'importation. 
: 
NATIONS UNTES

INSTITUT AFRICAIN

DE DEVELOPPEMENT ECONOMIQUE

ET DE PLANIFICATION

$\underline{D A} A \mathrm{R}$
IDEP/ET/VII/107

Cᄃ표 7

Avril 1964

Original : Anglais

TRADUCTION PROVISOIRE

D. Carney

\author{
RELATIONS ECONOMIQUES INTERNATIONALES
}

$7^{\circ}$ leçon : Rôle du Commerce International et des Investissements dans Ie Développement Economique : Commerce ou Aide

Pour les pays en voie de développement à économie ouverte.

$$
\begin{aligned}
& C+I=Y=C+S \\
& C+I_{d}+I_{f}=Y, \text { où } I_{f}=X-M \\
& C+I_{d}+X=Y+M=C^{\prime}+S+M
\end{aligned}
$$

Etant donié que pour les pays en voie de développement sans industrie de biens d'équipement, $X-\mathbb{M}$ peuvent raisonnablement être considérés comme négatifs (balance des paierents déricitaire des Jeunes Débiteurs).

Nous pouvons changer I'écuation $I_{f}=M-X$

Aussi $\mathrm{C}+\mathrm{I}_{\mathrm{d}}+\mathrm{M}=\mathrm{Y}+\mathrm{X}=\mathrm{C}+\mathrm{S}+\mathrm{X}$

où $M$ est de la même nature que $I_{d}$ et $X$ de la même nature que $S$.

Par conséquent, les invastissements bruts, intérieurs et étrangers peuvent être représentés par $\left(I_{\alpha}+M\right)$.

Mais 1.M ne représente pas uniquement les importztions en capitaux, une partie représente des importations de biens de consommation qui proviennent, de "l'effet de demonstration" international, ainsi que la tentative de diversifier les normes de la consommation et le niveau de revenu grâce aux importations de biens de consommation.

Par conséquent II comprend deux parties: biens de consommation et biens d'équipement.

$$
\begin{aligned}
& \text { Dès lors } M=M_{c}+M_{i} \\
& \text { Aussi } Y+\bar{X}=C+M_{c}+\bar{I}_{d}+M_{i}=\overline{C+S+X} \\
& \text { c.à.d. } Y=C+M_{c}+I_{d}+M_{i}=C+S+X \\
& Y \text { est le maximum } \\
& \text { lorsque } \frac{d Y}{d X}=0 \\
& \text { c.à.d. Iorsque } \frac{d C}{d X}+\frac{d M_{c}}{d X}+\frac{d I_{d}}{d X}+\frac{d M_{i}}{d X}=0=\frac{d C}{d X}+\frac{d S}{d X}+1
\end{aligned}
$$


Si l'on suppose que $I_{d}$ est un facteur indépendant dans la croissance de $Y$, et que ce facteur est constant, et aussi ágal à $S$ en équilibre.

$$
\begin{aligned}
& \text { Alors } \frac{d I_{d}}{d X}=0=\frac{d S}{d X} \\
& \text { et. } \frac{d C}{d X}+\frac{d M_{c}}{d X}+\frac{d M_{i}}{d X}=0=\frac{d C}{d X}+1 \\
& \text { c.à.d. } \frac{d C}{d X}=-1
\end{aligned}
$$

Par conséquent $\frac{d M_{i}}{d X}=1-\frac{d M_{C}}{d X}$

d'où $\frac{d M_{i}}{d \bar{X}}$ est plus grand lorsque $\frac{d M_{c}}{d X}$ est moindre ou zéro.

Il s'ensuit que l'effet d'investissement des importations sur

le volume de $Y$ en ce qui concerne les exportations, est le plus importanti lorsque l'effet de consommation des importations est moindre ou zéro.

Par conséquert : politique de restriction des importations de consommation on faveur d'importations d'investissements pour un apport maximum des importations aux investissements et à I'accroissement du revenu dans les économies ouvertes on voie de développement.

Commerce ou Aide?

$Y=C+I_{d}+(X)-M=C+S+(X)-M$

Si $X=0$ en tant que résultat de I'aide (M)

$\frac{d Y}{d M}=\frac{d C}{d M}+\frac{d I_{d}}{d M}-1=0=-\frac{d C}{d M}+\frac{d S}{d M}-1$

c.à.d. $\frac{d I_{d}}{d M}=\frac{d S}{d N}$

Autrement dit, le revenu $Y$ sera proté au maximum en raison de M, au cas où Ml provoque un taux égal de croissance en I et: $S$, c.è.d. Iorsque I'aide (M) conduit à un taux de croissance du volume de l'épargne intérieure équivalent au taux de croissance du volume des investissements intérieurs. Dans le simple cas de l'équilibre, lorsque $I=S$ cela signifie: en fait, que I'aide doit provoquer un montant égal de $S$ et de $I$ dans l'économie du pays bénéficiaire. Il dépendra de la nature et de la com.position de If que ce résultat se produise ou non. 
(1) Si M représente des importations de denrées alimentaires vendues pour(des fonds de contrepartie) qui sont investies par la suite dans des projets de développement dans le domaine de l'équipement, ceci devrait remplir la condition requise, mais a deux condistions supplémentaires :

(a) I'aide en aliments devrait se faire selon un calendrier et une méthode qui ne fait pas concurrence ou ne décourage pas la production intérieure et la vente de l'output de nature similaire aux produits alimentaires importés. Sinon, le résultat net de ces derniers serait réduit de manière correspondente.

(b) Il devrait être permis de dépenser les fonds de contrepartie pour l'importation de biens d'équipement en provenance du pays donateur - condition importante lorsque le pays bénéficiaire ne produit pas de biensd'équipement mais dépends des importations. Sinon, l'effet d'invostissemest de l'aide ne se réalise pas.

(2) SI M représente les importations de capitaux, la condition requise est remplie automatiquement dans le simple cas où Y n'est pas en équilibre.

Il découle des deux exemples, qu'une condition importante de l'aide étrangère remplaçant le commerce et contribuant à l'accroissement de la production dans un pays sous-développé avec une économie ouverte, est qu'une telle aide doit prendre la forme soit de bien d'investissement soit (directement ou indirectement) de fonds convertibles'en importations de biens d'équipement provenant du pays donateur. A cet égard l'aide sous conditions n'est pas un obstacle, sauf si le type de biens d'équipement importés, que le pays souhaite, ne pout pas être fourni par le donateur, mais par d'autres pays. C'est là une condition rigoureuse qu'il n'est pas facile de remplir dans un monde où 


\section{Page 4}

(a) I'aide est parfois fournie à des fins militaires et non pas pour le développement économique.

(b) I'aide prend souvent la forme de biens de consommation plutôt que d'équipement (assistance technique, personnel qualifié ou biens d'équipement, requis pour stimuler fortement l'économie.

(c) La plupart de I'aide contemporaine n'est pas permanente; elle est souvent accordée pour des raisons idéologiques plutôt que pour des raisons économiques. Par conséquent, il se peut qu'elles sont astreinte à des liens politiques invisibles ou à d'autres conditions.

(d) Pour les cas où I'aide prend la forme d'Assistance technique, c'est-àmire connaissances particulières ou personnel qualifié pour un projet d'équipement, il se peut qu'ils y ait les inconvénients suivants :

(i) il faudra trouver par la suite les biens d'équipethint par voie des arrangements commerciaux habituels pour que les connaissances acquises deviennent matériellement productives

(ii) le personnel technique ne travaillera peut-ôtre pas aussi $b$. en dans un milieu étranger, même muni de tout l'équipement nécessaire (et encore moins bien sans cet équipement) que dans son propre milieu

(iii) lorsqu'on n'utilise pas pleinement le personnel local, ceci peut donner lieu à la concurrence et au remplacement de personnel local également compétent par du personnel étranger, ou bien le personnel local qui devrait être adjoint au personnel étranger et être destiné à remplacer les experts, peut faire défaut.

Pour toutes ces raisons, les formes habituelles d'investissements commerciaux sont préférables aux investissements par l'aide. En particulier une transaction commerciale normale comprenant l'échange et les investisse.. ments par la voie du commerce présente les avantages suivants : 
(a) les investissements commerciaux normaux comprennent l'importation de biens d'équipement qui sont accompagnés de leur propre assistance technique

(b) ils peuvent ainsi court-circuiter les délais entre l'assistance technique et la mise en oeuvre du projet d'investissement

(c) Ie commerce implique des efforts é échॄnge日 réciproques conduisant à un accroissement de la richesse totale du monce alors qu'il n'en est pas ainsi dans le cas de l'aide; celieci donne quelque chose pour rien dans un sens matériel visible et redistribue simplement la somme totale de la richesse existante, sans I'augmenter.

Cependant, lorsque cela est possible, le Commerce et l'Aidc

doivent être envisagés oux fins de la croissance économique pourvu quo les conditions selon lesquelles l'aide peut accelérer la croissance au meximum soient satisfaites.

Jusqu'à présınt, les arguments en faveur du Commerce par préfé.. rence à I'Aice étaient basés sur les hypothèses suivantes :

(a) Le commerce est possible à des conditions mutuellement profitables entre les pays en voie de développement et le restie du monde, et toús particulièrement les pays industrialisés.

(b) Les investissements des pays industrialisés dans les pays en voie de développement sont possibles à une échelle suffisante pour permettre à ces derniers d'avoir un surplus permanent d'importations par rapport aux exportations de menière à promouvoir la croissance du jeune pays débiteur.

Pour autant que ces hypothèses soient exactes, l'argument pré-. cédent é faveur du commerce plutôt que de l'aide, ou les deux si possible, es.t valable.

Cependant, lorsque les hypothèses relatives à la profitabilité du commerca et à la possibilité d'investissements intexnationaux à long terme ne sont pas acceptables ou bien que de grandes réserves ou restrictions sont exprimées à leur endròit, on peut arriver aux conclusions opposées, donnant la préférence à l'aide sur le commerce. 
Page 6

Par conséquent, lorsque :

(a) Ie commerce des pays en voie de développement comprend des matières premières destinées à l'exportation dont les prix déclinent à long terme et pour lesquels la demande mondiale n'est.pas élastique, ce qui produit une déterioration des termes d'échange des pays en voie de développement (c.à.d. les pays industrialisés gagnentdarantage que les pays en voie de développement, même si tous deux y gagnent).

(b) Le flux international des investissements privés d'équipement diminue et est inadéquat face à la demande croissante d'un nombre toujours plus important de pays en voie de développement.

Il se peut que seule l'aide puisse faire sortir le pays de l'impasse. Ou bien encore, si les pays industrialisés ne sont pas en mesure d'augmenter leurs demandes aux producteurs de matières premières et si ces derniers ne peuvent restreindre leurs. ventes sur les marchés mondiaux afin de maintenir les prix, l'aide est peut-ôtre la seule alternative possible. Mais, dans ce cas, les conditions de l'utilisation au maximum de cette aide en vue de la promotion de la croissance ne devraient pas être perdues de vue. 
NATIONS UNIES

INSTITUT AFRICAIN

DEVELOPPEMENT ECONOMIQUE

ET DE PLANIFICATION

DAKAR
IDEP/ET/VII/ 107

8ème leçon

Original : anglais

Avril 1964

\section{LES RELATIONS ECONONIQUES INTERNATIONALES}

\section{Carney}

8ème leçon: Problèmes inhérents aux relations économiques internationales. Plans de stabilisation des prix et des produits.

1. Problèmes du prix des produits de base dans les pays en voie de développement.

1. Fluctuations des prix des produits de base - produits agricoles et minéraux (à l'exception de l'or, des diamants et des autres métaux précieux).

Exemple: - modèle oscillatoire de la toile d'araignée.

2. Fluctuations des recettes des producteurs de matières premières.

3. Fluctuations des revenus destinés aux projets de développement pour autant que ceux-ci dépendent fortement des gains provenant de la vente des matières premières sur le marché mondial.

4. Tendance à l'affaissement à long terme du prix des matières premières, en raison:

(a) du volume croissant de la production de matières premières

... (b) de la demande relativement inélastique pour les matièrés premières avec pour résultat l'affaissement des prix et des revenus alors que la production crôt.

(c) de la menace des produits synthétiques, par exemple les fibres, les substituts des métaux à la suite des.progrès techniques.

(d) Quantité décroissante de matières nouvelles dans les produits industriels.

2. Solutions proposées :

(a) Prix stables et garantis pour les matières premières.

Par exemple: les mesures prises par le Miarché Commun Européen pour garantir des prix stables pour les matières premières, los demandes de garanties deś prix similaires formuléés par les producteurs do matières premières et adressées aux pays 
industrialisés membres du GATT; les garanties données autrefois par les métropoles pour les matières premières écoulées par les offices de commercialisation coloniaux.

(b) Prix et volume de production garantis en vertu de plans internationaux de stabilisation des matières premières. Par exemple: ….......... l'étain, le projet le plus réussi jusqu'à présent sur le plan international.

(c) Financement compensatoire temporaire par le FMI des déficits dans la balance des paiements des pays producteurs de produits de base lorsque ces déficits sont dus à une mauvaise récclte ou à l'affaissement des prix sur le marché mondial.

(d) Plans de limitation de la vente des produits élaborés par les producteurs de matières premières.

3. Principes permettant d'évaluer les diverses propositions tendant à régler le problème des produits de base.

(a) Leur incidence sur les prix, dans le cas des plans de garantie, suivant que los prix mondiaux augmentent ou diminuent.

(b) Leur incidence sur la production.

(c) Leur incidence sur la répartition générale des ressources et sur l'utilisation des ressources.

4. Inconvénients des solutions proposées. Ce sont des remèdes provisoires et non pas des solutions à long-terme au problème des matières premières ou à la croissance industrielle.

(a) Plan's comportant la garantie des prix :

(i) ils ne sont pas nécessaires lorsque les prix mondiaux sont élevés et ils sont intolérables, et par conséquent d'une utilité limitée ou même nulle lorsque les prix mondiaux déclinent.

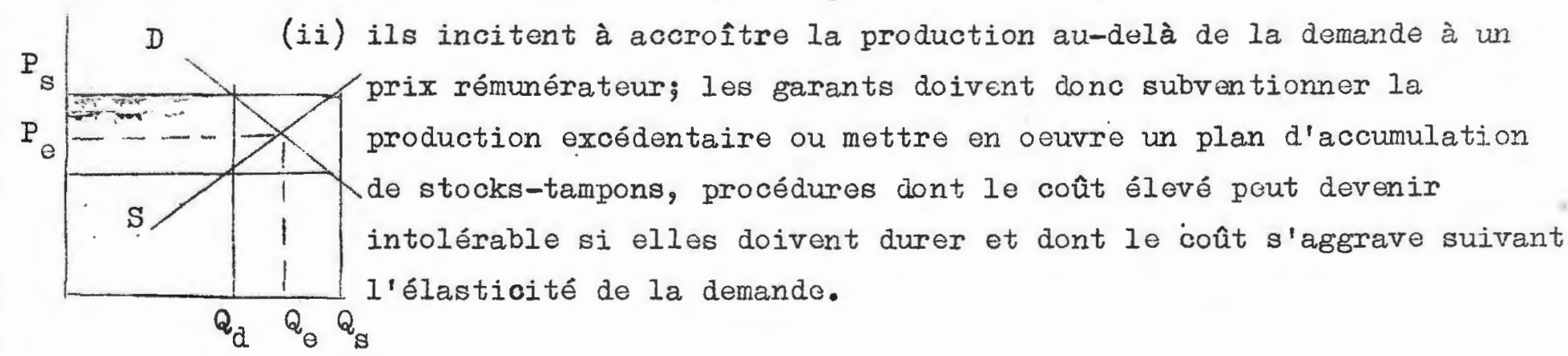


(iii) ils empêchent la diversification de l'utilisation des ressources ou le passage à de nouvelles productions, de nouvelles industries de transformation ou autres orientées vers le marché intérieur.

(iv) Au fur et à mesure du progrès technologique et l'apparition de prọduits de remplacement synthétiques qui en résulte, on voit grandir, l'écart technologique entre les pays avancés et les pays producteurs de matières premières qui n'ont pas appris à transformer leurs matières premières ou à les utiliser d'une autre manière.

(b) Garantie des prix et de la production:

(i) Les plans de ce type fixent des contingents aux producteurs, sur la base de la production moyenne d'une période donnée dans le passé, des amendes si ils dépassent le contingent ou s'ils ne le remplissent pas, et la redistribution. (tous les six moins environ) à d'autres producteurs du contingent des pays qui n'arrivent pas à le remplir.

(ii) Mécontentement de certains pays à propos de la répartition des contingents.

(iii) Accord international compromis si tous les principaux producteurs ne participent pas au plan et si tous les participants consommateurs ne s'engagent pas à ne pas acheter aux pays producteurs qui ne participent pas au plan.

(iv) Ce type de plan risque de décourager le passage - qui serait justifié à de nouveaux modes de production et la production en vue de la transformation et de la fabrication pour le marché intérieur.

(v) Suivant le volume de la production totale, déterminée par le conseil des producteurs et des consommateurs et suivant la demande des pays consommateurs, le plan tampon risque d'exiger des fonds considérables pour acheter lexcédent de la production sur la consommation pour le stocker pendant que les prix sont bas, et l'écouler lorsque les prix ont augmenté, ceci afin de stabiliser le marché.

(c) Financement compensatoire temporaire des déficits de la balance des paiements dos pays producteurs de matières premières.

(i) Ceci ne saurait être qu'une mesure temporaire ot non pas une solution définitive. 
(ii) Le FMI doit être convaincu que le déficit n'est pas' dû à une mauvaise politique financière de base, c'est-à-dire, à des mesures inflationnistes.

(d) Plans de limitation de la vente des produits:

(i) La difficulté consiste à arriver à un accord entre les principaux producteurs pour I'établissement d'un plan de ce genre.

(ii) C'est lo meilleur moyen de maintenir les prix à un niveau élevé sans avoir à lessubventionner directement, mais ce type de plan ne résoud pas le problème fondamental de la diversification des ressources et de I'utilisation des ressources.

5. Avantages des différents plans qui ont été proposés.

(i) En tant qu'expédients temporaires, ils permettent aux producteurs de matières premières de rechercher des solutions ayant un caractère plus définitif comme la transofrmation et l'utilisation industrielle des produits pour le marché intérieur outre les marchés mondiaux.

(ii) La meilleure justification de ces plans réside dans le fait que l'on peut utiliser les bénéfices qu'ils procurent à des investissements dans le secteur industriel afin que l'économie ne soit plus au même degré tributaire de la production ot de la vente des matières premières. 
NATIONS UNIES

INSTITUT AFRICAIN

DEVELOPPEINENT ECONONIQUE

ET DE PLANIFICATION

DA K A R
IDEP/ET/VII/107

Leçon 9

Original : anglais

Avril 1964

\section{RELATIONS ECONONIQUES INTERNATIONALES}

\section{Carney}

gème leçon : Problèmes inhérents aux relations économiques internationales. Système tarifaire et protection douanière, libéralisation du commerce, le GATT.

Systèmes tarifaires : Un système tarifaire est l'ensemble des droits perçus, sur les marchandises importées de l'étranger, pour s'assurer des recettes ou pour protéger les produits locaux/dans les deux intentions à la fois.

(a) Recettes : si au droit d'importation correspond un droil de régie équivalent sur la production locale.

(b) le système est protecteur si les droits sont si élevés qu'ils interdisent toute importation.

(c) il assure à la fois des recettes et la protection des produits locaux si le droit d'importation n'est pas prohibitif et s'il n'y a pas de droit de régie correspondant sur la production locale.

Note : Si on perçoit un droit d'importation sur un produit importé qui ne figure pas dans la production locale, I'effet est protecteur, dans la mesure où la demande est détournée vers d'autres produits locaux ou importés. Un produit (i) qui n'est pas produit localement, (ii), dont la demande n'est pas très élastique, et (iii) dont la consommation locale est importante est un produit idéal si l'on désire se procurer des recettes.

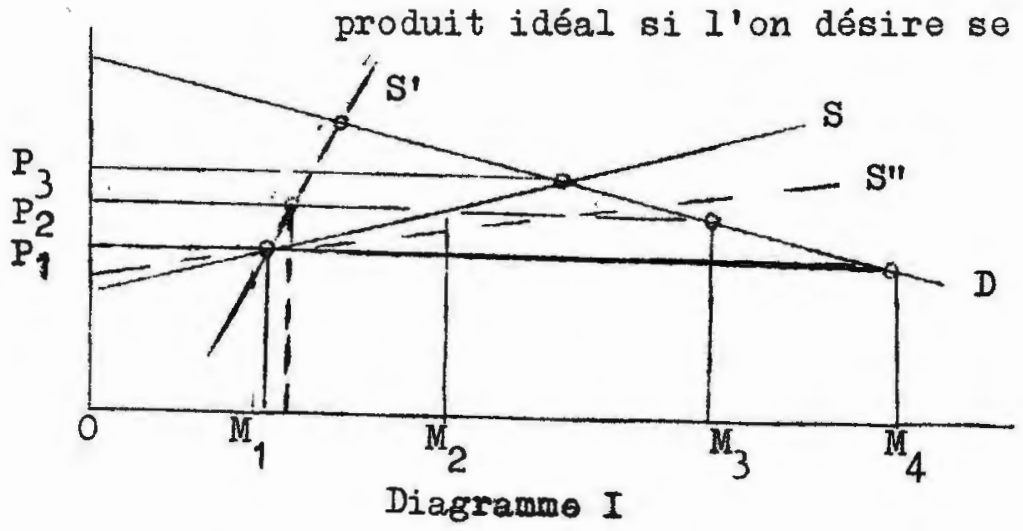


Note : Les courbes $S$ et $D$ étant données,

(i) $P_{1} P_{3}$ est un système tarifaire protectour pur et simple, qui interdit l'importation du produit.

(ii) $P_{1} P_{2}$ eśt en partie protecteưr, en partie fiscal (destiné à produire des recettes).

Les courbes $S^{i}$ et $D$ étant données, (iii) $P_{1} P_{2}$ est plutôt fiscal que protecteur.

Les courbes $S^{\prime \prime}$ et $D$ étant données, (iv) $\mathrm{P}_{i} \mathrm{P}_{2}$ est plutôt protecteur que fiscal.

Par conséquent, pour une courbe donnée de la demande, l'importance relative des ếets protecteurs et fiscaux dépendra du degré d'élasticité de la courbe de l'offre. Si la courbe de l'offre est relativement plus élastique, l' jifet protecteur est plus important que l'effet fiscal. Si la courbe de l'offre est relatirement moins élastique, c'est le contraire. Dans le promier cas, les productcins locaux ont une part plus importante extérieur marché

Un système tarifaire qui protège une production locale dont le coût va croissant ne peut donc être efficace que si la courbe de l'offre est relativement élastique. c'est-à-dire si les coûts de la production locale ne montient que petit à petit.

Note : $\mathbf{t}=$ recettes supplémentaires'pour les producteurs locaux, sur la production antérieur:.

$p=$ effet de protection (recettes) pour les producteurs locaux sur la production protégée.

$r$ = effet fiscal pour le gouvernement.

$c=$ pertes non compensées pour les consommateurs

$p+o=$ perte totale non compensée des consommateurs en plus de la quantité, $\mathrm{M}_{3} \mathrm{M}_{4}$, prévue par les consommateurs.

Un tarif protecteur ne se justifie que dans le cas où le coût moyen de la production est en baisse. Un tarif protecteur peut être efficace lorsque les coûts de production augmentent, mais impliquerait une mauvaisé répartition ou une utilisaticn inefficace des ressources marginales tranfférées à la production sur le marché intérieur en comparaison (a) d'autres utilisations possibles, (b) du co.t de la même production sur le marché international. 
Le fait que la production dans des conditions de coûts croissants de production aboutit à la spécialisation partielle et s'accorde avec le libre échange, n'atteint pas l'argument suivant lequel la protection, dans ces circonstances, peut entraîner une répartition et une utilisation relativement inefficaces des ressources marginales intérieures.

Libre échange : Arguments "pour" (dans l'hypothèse d'un coût de production en accroissement).

Si le commerce $n^{\prime}$ est pas soumis à un tarif douanier, il revient moins cher de se procurer les biens et services à l'étranger que de produire localement des produits substituables, et l'on peut orienter vers des types de production plus =entables les ressources qui ne sont pas employées à la production de produits substituables.

Arguments contre la protection douanière (dans l'hypothèse où le coût de la production augmente).

(1) Elle fait passer lcr ressources d'un meilleur emploi à un emploi moins efficace.

(2) En faisant monter les prix des biens et services, elle réduit la liberté de chsix du consommateur, et le contraint arbitrairement à réduire sa consommation de biens qu'il achèterait en plus grande quantité s'il avait toute liberté de choix.

Arguments contre le libre échange et pour la protection.

(1) Seulement dans les cas où la production de la marchandise implique des coûts de production décroissants et peut-être des coûts et des prix inférieurs à ceux des concurrents qui se sont trouvés les premiers dans le domaine en vertu d'un accident de.l'histoire; dans ces cas làg il est justifié d'utiliser la protection douanière pour remédier au déséquilibre d'origine purement accidentelle.

(2) La protection douanière peut également avoir un effet sur la répartition du revenu entre les différents groupes économiques du pays, par exemple, en protégeant l'agriculture, on réserve le marché intérieur aux producteurs locaux, ce qui peut accrôtre la part du reveriu national qui revient aux fermiers en comparaison de celle qui revient au secteur de fabrication (cas des Etats-Unis). Mais on 
obtient ce résultat au prix diune mauvaise affectation des facteurs.

Les arguments pour le libre échange sont valables si les conditions suivantes sont réalisées.

(1) Coûts de production croissants.

(2) Mobilité des facteurs si les techniques sont en progrès rapide et si les fonctions de production (ou les combinaisons cies facteurs) autres que celles qui tiennent aur dons de la valeur sont en évolution. Pour des raisons d'économie nationale pár exemple, pour protéger le niveau de l'emploi, et parce que 7.a mobilité de l'utilisation des ressources exige souvent le recyclage des facteurs de production (an particulier de la main-d'oeuvre), alors que, en même temps le capital fixe est moins mobile entre les diverses utilisations, le concept du libre échange échoue dans la pratique et devient inapplicable.

On a aussi fait remarquer que les arguments en faveur du libre échange ne sont séduisarts qu'en l'absence de concurrence industrielle sérieuse, comme ce fut le cas pour la Grande Bretagne au 18ème siècle et au début du 19ème sièclẹ mais ils tombent devant la concurrence industrielle extérieure, comme ce fut le cas de la Grande Bretagne à la fin du 19ème siècle et au début du 20ème siècle lorsque les ttats-Unis, l'Allemagne et le Japon lui firent concurrence.

\section{QUELQUTS ARGUWENTS EN FAVEUR DE LA PROTECTION DUU 1NIERE :}

(a) Diversification des industries - Le cas de l'indu: trie maissante:

Etant donné que la production lorsque les coûts vont croissant est compatible avec la spécialisation partielle et le libre échange, on peut conseilier la protection aux pays dont les exportations sont fondées sur un petit rombre de matières premières, afin de leur permettre de diversifier leur économie. Dans ces cas là, il serait bon d'assortir la protection lie mesures destinées à réduire les coûts de production et par conséquent à permettre aux industries protégées de survivre sans protection. 
Si cette condition ne peut être remplie, il peut devenir définitivement inefíicace de détourner les ressources vers les industries protégées.

(b) Tarifs en vue de négociations :

Les tarifs peuvent être utilisés en tant qu'instrument de pression pour pousser les autres pays à abaisser leurs tarifs dans I'intérêt réciproque et à élargir ainsi la zone du libre échange (ex. : Le Kennedy Round de la réduction des tarifs sur les produits industrielsy 1964).

Cependant, ceci peut échouer si les industries et les intérêts protégés s'opposent à la suppression des tarifs sous prétexte qu'il leur est impóssible de réaffecter leurs ressources.

(c) Tarifs anti-dumping

Ie dumping se léfinit comme la descrimination des prix sur la base des différences entre l'élasticité de la demande sur le marché intérieur et sur les marché extérieur, tout spécialement lorsque le prix de demande est plus bas sur le marché extérieur que sur le marché marché intérieur, compte tenu des frais de transport, des taxes et des autres frais.

C'est une bonne méthod: économique

dont l'idée est d'égaliser les revenus marginaux sur les deux marchés. Mais, les résultats dépendent, en grande partie, des coûts de production des ventes combinées sur les deux marchés. Il se peut fort bien que le coût de production plus tous les autres frais de transfert fassent un prix extérieur inférieur au prix intérieur. La descrimination des prix serait encore justifiée.

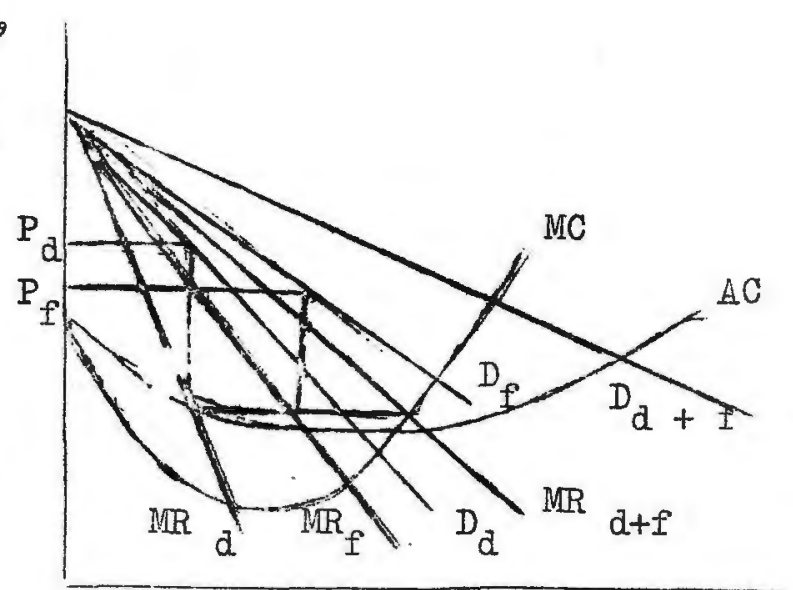

Diagrammo II 
Ce n'est que dans le cas où le prix extérieur est inférieur aux coûts de production, y compris les frais de transfert au marché extérieur que l'on peut soulever une objection fondée (i) sur le fait que le résultat représenterait une répartition peu économique des ressources par le vendeur, car les ventes se font à un prix inférieur au coût, өt (ii) sur le fait que de telles tactiques de vente ruinent l'industrie concurrentielle du marché importateur.

Si l'on peut démontrer que ces raisons existent les taxes "anti-dumping" peuvent se justifier. Sinon, les droits"anti-dumping" simplement fondés sur la discrimination des prix,peuvent servir de prétexte pour entraver le commerce et priver le marché de l'avantage d'une demande fortement élastique. En effet, étant dcnné que le dumping en dessous du coût augmenté des frais de transfert peut être difficile à prouver, il n'y a aucun doute, que bien souvent, lorsqu'un producteur se plaint d'avoir été lésé par le dumping, ce n'est qu'une excuse pour justifier son incapacité à faire face à la concurrence internationale ou un moyen de protéger le marché intérieur pour des raisons nationalistes.

(d) Amélioration des termes de I'échange.

Les droits perçus sur les produits importés peuvent être un moyen d'obliger le pays producteur à baiss эr le prix de l'importation s'il désire échapper à la barrière douanière. Ceci, automatiquement, assure de meilleurs termes d'échange au pays importateur. Mais une grande partie de ces avantages dépend :

(i) des conditions de coût du pays exportateur - parfaitement élastiques, c'est-à-dire que des coûts sonstants ou presque constants rendront le tarif inutile, mais $\mathrm{k}$ : les coûts sont en augmentation ou en diminution, il peut être utile en abaissant le niveau des coûts en augmentation et en activant la baisse des coûts ou en explorant les possibilités de baisse offertes.

(ii) possibilité de rétorsion; dans ce cas l'issue est incertaine et il est même possible que les partịes commerçantes se retrouvent avec un volume réduit de transactions sans qu'il y ait de modifications perceptibles dans les termes de l'échange. 
(iii) la production de produits substituablas dans des conditions de coût croissant peut renare inefficace la réaffectation des ressources dans le pays qui impose les droits.

(e) Protection du niveau de l'emploi sur le marché intérieux. (exportations du chômage).

Surtout dans les périodes de crise comme celle des années 30 , on peut utiliser cet argument en faveur des tarifs dans le but essentiellement nationaliste de renforcer l'emploi et la demande intérieurs. Ceci est conformo à l'analyse de Keynes et-à la USA $/$ Oefré Royaume-Un: doctrine du multiplicateur du commerce eztérieur seinn laquelle les exportations créent des revenus et favorisent le plein emploi alors que les imporiations produisent l'effet contraire.

Cependant, cet objectif peut ne pas Ǵtre atteint car, même si Ies autres pays ne

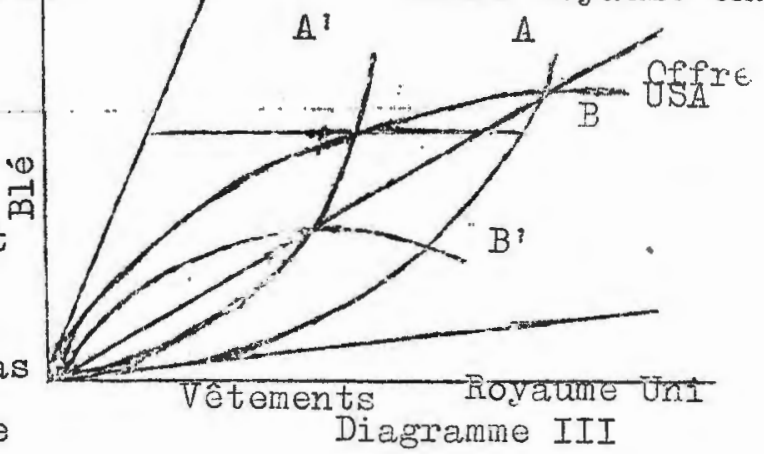
preiment pas de mesures de représailles lorsqu'un pays A réduit ainsi les importations en provenance d'un pays $B$, le pays $B$ est moins ̀̀ même d'achete: les exportations du pays $\dot{H}$, si bien que le chômage qui avait été exporté par le pays A reviont s'installer à demeure. Il est peut-être préférable de chercher à maintenir le niveau de l'emploi par l'expansion monétaire et le contingentement plutôt que par la protection douanière.

(f) Ia défense nationale (argument non-économique).

Iorsqu'on prévoit la guerre, la protection douanière peut stimuler le progrès technique, les recherches qui se rapport nt à la défense et la production de substitu's accroissant ainsi l'autonomie du pays condition fort importarte poun gagner une Euerre.

Cependant, (i) à moins de donner une définition très étroite du concept de défense nationale, ce concept peut recouvrir toutes les industries, auquel cas il faudrait protéger massivement toute l'économie (Allemagne de 1939 à 1945), avec pour résultat une mauvaise répartition générale des ressources. 
(ii) Si le concept est défini de façon étroite et appliquée seulement à quelques industries et à quelques produits clés, il est préférable que le Gouvernement entreprenne de produire directement pour ses propres besoins ou qu'il accorde des primes ou des subventions aux entreprises privées qui produisent ces marchandises.

\section{ARGUMENTS SANS VALEUR EN FAVEUR DE LA PROTECTION •}

1. Empêcher la sortie de l'argent : Dans le commerce international ce sont ies biens et services qui servent à payer les biens et services; les monnaies nationales ne sont utilisée que dans leur pays d'origine et non pas à I'étranger. Cependant, c'est un argument du même type que celui qui veut "empêcher l'emploi de sortir" et il connất les mêmes limites.

2. Nivellement des coûts de production sur le marché intérieur et extérieur. Les tarifs dits "scientifiques".

(i) En fait, se ne sont pas les coûts qui sont nivelés sur les deux marchés, car ils restert différents, mais o'est le prix du produit dans les deux pays, compte tenu du transport et des autres frais de transfert. En ajoutant ces frais, les prix dans les deux pays doivent être différents, à moins qu'il y ait discrimination des pix sur les deux marchés.

(ii) Soul le libre échange peut égaliser las coûts d'une manière durable et utile.

(iii) On ne sait pas comment les coûts sont égalisés dans la pratique, ni comment il faut choisir sur le marché extérieur, le produit, I'entreprise ou I'industrie dont il serait bon d'égaliser les coûts.

(iv) Le résultat est d'encourager une production inéfficace sur le marché intérieur. 
Ils produisent le même résultat que les tarifs à condition qu'on connaisse bien les courbes de I'offre et.de la demande pour le produit en question et que celles-ci ne soient pas complètement inélastique. Les résultats sont les mêmes en ce qui concerne la hausse des prix internes, la limitation du volume des importations et l'encouragement à la production locale, avec cependant les différences suivantes :

(a) Les termes de l'échange sont indéterminés - ils peuvent être plus ou moins favorables au pays qui impose les contingents et il n'est pas possible de prévoir ce qui sera le cas.

(b) Ceux qui bénéficient de la hausse du prix du produit dont l'importation a été limitée peuvent être soit les importateurs ou exportateurs, suivant qu'ils possèdent ou non le monopole du commerce de ce produit; soit le gouvernement qui en accordant des licences d'importation au plus offrant peut tirer des recettes de cette hausse des prix, comme cela se passe pour les tarifs.

Il y a trois motifs principaux de co tingentement :

(1) La certitude : Si I'on ne connaît pas les courbes de l'offre et de la demande, et du fait de la politique des prix des pays exportateurs, il peut être difficile de prévoir quelles seront les incidences des tarifs et quel devrait être leur ordre de grandeur pour éviter qu'une crise venue de I'extérieur envahıs, o le pays. Il est plus facile de prévoir les résultats du contingentement.

(2) I'inélasticité de l'offro extérieure de produits importés permet d'augmenter le prix du produit local en fixant un contingent assez faible aux importations.

(3) Ia souplesse : En cas d'urgence le système des contingents est plus souple que le système tarifaire qui devient généralement une institution du fait d'accords commerciaux avec clause de la nation la plus favorisée et d'autres limitations de la liberté d'action. Il n'est donc pas facile de changer les tarifs. Ils sont utiles lorsque les conditions économiques sont bonnes, mais non pas lorsqu'elles sont mauvaises, (par exemple en temps de crise). 
